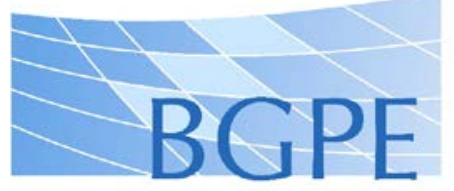

BGPE Discussion Paper

No. 170

\title{
Effects of Macroeconomic Uncertainty and Labor Demand Shocks on the Housing Market
}

\author{
Gabriel Lee \\ Binh Nguyen Thanh \\ Johannes Strobel
}

June 2017

ISSN 1863-5733

Editor: Prof. Regina T. Riphahn, Ph.D.

Friedrich-Alexander-University Erlangen-Nuremberg

(c) Gabriel Lee, Binh Nguyen Thanh, Johannes Strobel 
June 16, 2017

\title{
Effects of Macroeconomic Uncertainty and Labor Demand Shocks on the Housing Market*
}

\begin{abstract}
This paper shows that macroeconomic uncertainty affects the housing market in two significant ways. First, uncertainty shocks adversely affect housing prices but not the quantities that are traded. Controlling for a broad set of variables in fixed-effects regressions, we find that uncertainty shocks reduce both housing prices and median sales prices in the amount of $1.4 \%$ and $1.8 \%$, respectively, but the effect is not statistically significant for the percentage changes of all homes sold. Second, when both uncertainty and local demand shocks are introduced, the effects of uncertainty on the housing market dominate that of local labor demand shocks on housing prices, median sell prices, the share of houses selling for loss, and transactions. The aforementioned effects are largest for the states that exhibit relatively high housing price volatilities, suggesting real options effects in the housing market during the times of high uncertainty.
\end{abstract}

- JEL Classification: E4, E5, E2, R2, R3

- Keywords: Bartik labor demand shocks; time-varying uncertainty shocks; real options effects; housing market.

Gabriel Lee (Corresponding author)

University of Regensburg

Universitaetsstr. 31, 93053 Regensburg, Germany

And

Institute for Advanced Studies

Josefstaedterstr. 39, 1080, Wien, Austria

gabriel.lee@ur.de, + 499419435060

Binh Nguyen Thanh

University of Regensburg

Universitaetsstr. 31, 93053 Regensburg, Germany

binh.nguyen-thanh@ur.de, + 499419432739

Johannes Strobel

University of Regensburg

Universitaetsstr. 31, 93053 Regensburg, Germany

johannes.strobel@ur.de,+ 499419435063

\footnotetext{
${ }^{*}$ We thank the seminar and conference participants at the 2016 Asian Econometric Society Meeting, 2016 European Real Estate Society Meeting, 2016 Asian Real Estate Society Meeting, the Econometric Seminar at the University of Regensburg and the Bavarian Graduate Program in Economics for constructive comments. Gabriel Lee and Johannes Strobel gratefully acknowledge financial support from the German Research Foundation ((DFG) LE 1545/1-1).
} 


\section{Introduction}

Three well-documented features of the recent Great Recession are the decline in housing prices, the increase in unemployment rate, and the increase in the presence of uncertainty in the U.S. Figure 1 shows the correlations between the U.S. housing price growth rate and some of the uncertainty measures that are in the recent literature: a clear negative correlation between the housing price growth rate and the shown uncertainty measures. ${ }^{1}$

Figure 1 here

Figure 2 also shows a strong negative correlation between the monthly U.S. unemployment rate and the Bartik index that proxies the U.S. labor demand shocks from 1990 to 2014.

Figure 2 here

The objective of this paper is to examine the simultaneous effects of macroeconomic uncertainty - and local labor demand shocks on the U.S. housing market. ${ }^{2}$ More precisely, we seek to answer (i) does an uncertainty shock directly affect the housing market, (ii) if a local labor demand shock occurs in a period of high uncertainty, is the impact different compared to a period of low uncertainty and (iii) how robust are the outcomes given the choice of the uncertainty proxy and the threshold level defining a period of high uncertainty? Our paper adds to the growing number of recent papers that deal with the effects of uncertainty - and labor demand shocks on an aggregate economy as well as housing and labor markets. But our approach differs from others as we analyze the simultaneous effects of both shocks on the U.S. housing market. For example, Christiano, Motto and Rostagno (2014), Mehkari (2016) or Berger, Grabert and Kempa (2017) show that uncertainty adversely impacts the aggregate economy, while Dorofeenko, Lee and Salyer (2014) show uncertainty shocks can explain the U.S. housing price volatilities. For

\footnotetext{
${ }^{1}$ We use four different uncertainty measures in our analysis: the macroeconomic uncertainty by Jurado, Ludvigson and $\mathrm{Ng}$ (2015) (Macro Uncertainty), the VIX by Bloom (2009), the economic policy uncertainty by Baker, Bloom and Davis (2016) (Policy Uncertainty), and our measure, which is analogous to Baker et al. (2016) but on a state level (State Uncertainty). Correlations between these uncertainty measures over these periods range between 0.25 and 0.63 .

${ }^{2}$ We specifically look at the average housing prices, the median selling prices, the share of houses selling for loss and transactions (houses sold).
} 
the labor demand shock on housing and labor markets, Edlund, Machado and Sviatschi (2016) examine the impact of labor demand shocks, using the Bartik index, on housing prices, and Shoag and Veuger (2014) empirically show that uncertainty may amplify labor demand shocks.

Controlling for a broad set of variables in fixed-effects regressions, our empirical results are as follows. First, we find that uncertainty shocks directly affect prices but not quantities. Both the median sale price and the housing price decrease on average by $1.80 \%$ and $1.42 \%$, respectively, but the effect is not statistically significant for the percentage changes of all homes sold. Second, a positive local labor demand shock significantly increases median sale prices, house prices, and transactions, and decreases the share of houses selling for a loss. If a labor demand shock occurs during a period of high uncertainty, however, then it essentially affects neither prices nor quantities: Home sellers and -buyers do not trade at the price and wait out in selling and buying until the uncertainty periods are over. This observation is consistent with the occurrence of a real options effect akin to the irreversibility of an investment described by Pindyck (1991, p.1117): "There will be a value to waiting (i.e., an opportunity cost to investing today rather than waiting for information to arrive) whenever the investment is irreversible and the net payoff from the investment evolves stochastically over time". For instance, Bloom, Bond and Van Reenen (2007) show that because of real options effects, firms' responsiveness to demand shocks is generally lower in periods of high uncertainty. The real option effects on real estate have also been documented by, for example, Capozza and Helsley (1989), who examine the impact of uncertainty on land values and development decisions in a spatial context. ${ }^{3}$

Analogous to the irreversible investment literature, we find the response of housing market variables to labor demand shocks to be much lower in times of high uncertainty, suggesting real options effects (option to "wait and see") in the housing market during the times of high un-

\footnotetext{
${ }^{3}$ Other representative papers on real option and real estate are Childs, Riddiough, and Triantis (1996), who demonstrate that the ability to mix uses and to redevelop affects the timing of land development, while Holland, Ott, and Riddiough (2000), Childs, Ott, and Riddiough (2002), Clapp, Eichholtz, and Lindenthal (2013), Bulan, Mayer, and Somerville (2009), and Cunningham (2006, 2007) empirically show that real options play an important role in house price dynamics, housing investment and land prices.
} 
certainty. More specifically, we show that following an adverse shock in labor demand of one standard deviation, the real option value ("wait and see" effect) in the housing price amounts to $0.19 \%$, and the effect increases to $0.32 \%$ for the states (locations) that exhibit relatively high housing price volatilities. We further find that following an adverse labor demand shock, the share of houses selling for loss significantly decreases in times of high uncertainty, but the number of homes sold remains almost constant. ${ }^{4}$ To further support of our hypothesis that the real option value increases with higher uncertainty, we sort the fifty one states into three equal-sized groups, according to the unconditional housing price volatility in each state. In doing so, we find that while the impact of local labor demand shocks is largest for the group with the highest housing price volatility, uncertainty completely offsets the labor demand shock - as opposed to the other two groups, where we find no significant impact of uncertainty.

We address real option issues in housing markets using monthly U.S. state-level data from 1990 to 2014. We construct binary uncertainty dummies to indicate the periods of high uncertainty, as in Bloom (2009) and a variation of Bartik (1991) index as local labor demand shocks to quantify the impact of these two shocks on the housing market. Our approach thus corresponds to models using two-state Markov-switching processes, where regime changes can be documented by an uncertainty index crossing various threshold values, which are based on the percentiles of the distribution of the uncertainty proxy. ${ }^{5}$

Our results, thus, indicate uncertainty shocks affect housing price movements both directly and indirectly. On the one hand, uncertainty adversely affects housing prices. On the other hand, uncertainty alters the impact of local labor demand shocks during uncertain times. With this latter effect being consistent with the presence of real option effects arising in a period of high

\footnotetext{
4 We show the robustness of the above results to different threshold values that are ranged from 80 th, 85 th, 90th and 95th percentile of an uncertainty proxy.

5 Our approach in defining the threshold values differs from the one used in, for example, Bloom (2009), who defines periods of uncertainty as the proxy when 1.65 or more standard deviations above the mean. We use the Macro Uncertainty measure by Jurado et al. (2015) as our benchmark measure but we also include other uncertainty measures such as the Policy Uncertainty proxy by Baker et al. (2016), the VIX which is also used by Bloom (2009), and the State Uncertainty similar to Baker et al. (2016) to analyze the state level housing markets.
} 
uncertainty in the housing market. ${ }^{6}$ One important implication of our results, analogous to Bloom et al. (2007), is that in order for policy measures to work properly, highest priority should be given to the reduction of uncertainty. ${ }^{7}$

\section{Data, Bartik Index and Uncertainty Measures}

In the following section, we describe the data as well as the construction of the Bartik index and various uncertainty measures used in our empirical analysis.

\subsection{Data}

We use monthly state-level data from 1990:1 to 2014:12; the data and sources are described in detail in the Appendix. Zillow Real Estate Research data and Freddie Mac provide information on various aspects of the housing market, such as the housing price, median sales price, the share of houses sold for loss and turnover. The housing price is the inflation adjusted housing price index from Freddie Mac; the median sales price is defined as the median of the selling price for all homes sold in a given state. The share of houses sold for a loss is defined as the percentage of homes in an area that sold for a price lower than the previous sale price and turnover is defined as the percentage of all homes in a given area that is sold in the past 12 months. These housing variables constitute the vector of dependent variables.

\subsection{Bartik Index: Labor Demand Shock}

The Bartik index is a measure of the predicted change in demand for employment in a state given by the interaction between a state's initial industry mix and national changes in industry employment.

The Bartik variable is a weighted average of economy-wide employment shifts, where the weights

\footnotetext{
${ }^{6}$ See also Aastveit, Natvik and Sola (2013), in which structural Vector Autoregressions are used to document wait-and-see effects in monetary policy during periods of high uncertainty. See also Bloom (2014) for further discussion and sectors where real option effects arise.

${ }^{7}$ Especially in light of the results of Stroebel and Vavra (2015), who show that there is a causal relation between changes in housing prices and changes in retails prices and thus consumption.
} 
reflect the relative fraction of local employment in each of the sectors. More specifically, the index compares the preexisting differences in the sectoral composition of employment across states with the broad changes in national employment, especially changes subject to a trend, asymmetrically impact states. Consequently, we use the Bartik index as a proxy for a labor demand shock. In this paper, we follow Saks (2005) to construct the Bartik index:

$$
\operatorname{Bartik}_{i t}=\sum_{j} \frac{e_{i j t-1}}{e_{i t-1}}\left(\frac{\tilde{e}_{i j t}-\tilde{e}_{i j t-1}}{\tilde{e}_{i j t-1}}-\frac{e_{t}-e_{t-1}}{e_{t-1}}\right)
$$

where $i=$ state, $j=$ industry, $t=$ month; $\tilde{e}_{i j t}=$ national industry employment outside of state $i ; e_{i t}=$ state employment $=\sum_{j} e_{i j t} ; e_{t}=$ national employment $=\sum_{i} e_{i t}$.

The first fraction reflects the share of industry $j$ employment relative to the total employment in state $i$ in $t-1$, the second fraction is the growth rate of industry $j$ outside of state $i$ and the third fraction reflects the change in national employment. Thus, the term in brackets reflects the change in industry $j$ employment (outside state $i$ ) relative to changes in national employment. This term is weighted by the "importance" of industry $j$ in state $i$ in $t-1$. We use $j=4$ sectors across $i=51$ states in this analysis: manufacturing, private services, public services and construction and logging. We use the time series of the Bartik index aggregated across states as displayed in Figure 2. The results remain unchanged if we exclude the construction sector from the Bartik index.

\subsection{Uncertainty Measures}

Various uncertainty proxies have been proposed in the recent literature. As shown in Figure 1, depending on the preferred proxy, the number of uncertainty shocks may differ considerably, although it is also possible that different proxies capture different aspects of uncertainty. We use the Macro Uncertainty measure, due to Jurado et al. (2015), for our baseline results because it captures the overall macroeconomic uncertainty and it is, by construction, uncorrelated with any single time series. 
Macro Uncertainty $U_{t}^{y}(h)$ builds on the unforecastable components of a broad set of economic variables. Jurado et al. (2015) estimate Macro Uncertainty as the conditional standard deviation of the purely unforecastable component of the future value, which translates to removing the forecastable component of a multitude of aggregated and weighted financial and real variables before calculating their conditional standard deviation. More specifically, they calculate for 132 macroeconomic time series $y_{j t} \in Y=\left\{y_{1 t}, \ldots, y_{132 t}\right\}$ the conditional standard deviation of the unpredictable component of the $h$-step-ahead realization:

$$
U_{j t}^{y}(h)=\sqrt{E\left[\left(y_{j t+h}-E\left(y_{j t+h} \mid I_{t}\right)\right)^{2} \mid I_{t}\right]}
$$

with $E\left(. \mid I_{t}\right)$ the expectations taken conditional on information $I_{t}$. Then, they aggregate these unpredictable components to obtain

$$
U_{t}^{y}(h)=p \lim _{N_{y} \rightarrow \infty} \sum_{j=1}^{N_{y}} w_{j} U_{j t}^{y}(h)
$$

with $w_{j}$ the aggregation weight. To compute $U_{j t}^{y}(h)$, Jurado et al. (2015) first form factors from a large set of economic and financial indicators, which represent the available information at time $t, I_{t}$. These factors are used to approximate the forecastable component $E\left(y_{j t+h} \mid I_{t}\right)$ and to calculate the forecast error $E\left[\left(y_{j t+h}-E\left(y_{j t+h} \mid I_{t}\right)\right)^{2} \mid I_{t}\right]$. Then, they estimate a parametric stochastic volatility model for the one-step ahead prediction error to obtain the conditional volatility the conditional variance of this error, $E\left[\left(y_{j t+h}-E\left(y_{j t+h} \mid I_{t}\right)\right)^{2} \mid I_{t}\right]$. Given these estimates, $h$-step ahead prediction errors can be calculated recursively. Finally, the individual forecast errors are aggregated, using equal weights $w_{j}$ for each time series $U_{j t}^{y}(h)$. For our results, we use the one-step ahead prediction error.

We also use three other uncertainty measures for the robustness check on our empirical analysis. First, the VIX measures the expected volatility of the S\&P 500 index and is the square root of the sum of squared standard deviations of the $\mathrm{S} \& \mathrm{P} 500$ rate of expected returns for the 
next 30 days. More technically, the VIX is the square root of a weighted average of the forward prices of out-of-the-money put and call options and approximates the price of a portfolio of options that replicates the payoff on a variance swap. Second, the economic policy uncertainty (Policy Uncertainty) measure proposed by Baker et al. (2016) proxies for movements in policyrelated economic uncertainty. The index quantifies the frequency of articles in 10 leading U.S. newspapers that contain the following triple of words: "economic" or "economy"; "uncertain" or "uncertainty"; and one or more of "congress", "deficit", "Federal Reserve", "legislation", "regulation" or "White House". Third, the state-level uncertainty indicator is constructed as the monthly number of newspaper articles in a state containing either one of the keywords "economic uncertainty", "economy uncertain" or "economy uncertainty" from 2000:1 until 2014:12 from the homepage www.newslibrary.com. ${ }^{8}$ In creating this index, we follow Baker et. al (2016). As can be seen in Figure 1, there are considerable differences in fluctuations, and thus in the periods classified as uncertain. ${ }^{9}$

A definition of the threshold value is needed in order to identify the number of uncertainty periods and to construct binary uncertainty series. Bloom (2009) suggests using "1.65 standard deviations above the mean, selected as the $5 \%$ one-tailed significance level treating each month as an independent observation". However, specifying the threshold in this manner does not leave any adjustment opportunity if the assumption of Normality and independently and identically distributed uncertainty shocks does not hold. ${ }^{10}$ Table 1 shows the number of months defined as "uncertain" by various uncertain proxies.

For example, using the Macro Uncertainty measure of Jurado et. al (2015), when $\alpha$ equals $5 \%$ then the Normal Distributional assumption leads to seventy-six uncertain periods instead of fiftyeight periods when one uses the corresponding percentiles of the actual distribution. Consequently,

\footnotetext{
${ }^{8}$ We also scale the State Uncertainty indicator by the number of newspapers and normalize it by dividing by the standard deviation in each state.

${ }^{9}$ See Strobel (2015) for further elaboration on the reasons for this observation.

10 We tested for the normality of the uncertainty proxies using the Jarque-Bera test, and the null of normality was rejected for each proxy.
} 
Table 1: Number of months defined as uncertain.

\begin{tabular}{l|l|l|l|l|l|l|l|l}
\hline & \multicolumn{2}{|c|}{$20 \%$} & \multicolumn{2}{c|}{$15 \%$} & \multicolumn{2}{c|}{$10 \%$} & \multicolumn{2}{c}{$5 \%$} \\
\hline \hline & $1-\alpha$ & $\alpha$ & $1-\alpha$ & $\alpha$ & $1-\alpha$ & $\alpha$ & $1-\alpha$ & $\alpha$ \\
& Percentile (P) & Normal (N) & $\mathrm{P}$ & $\mathrm{N}$ & $\mathrm{P}$ & $\mathrm{N}$ & $\mathrm{P}$ & $\mathrm{N}$ \\
Macro & 124 & 104 & 103 & 96 & 80 & 86 & 58 & 76 \\
Policy & 192 & 188 & 174 & 175 & 156 & 162 & 138 & 148 \\
State & 36 & 27 & 27 & 21 & 18 & 18 & 9 & 13 \\
VIX & 240 & 222 & 225 & 217 & 210 & 206 & 195 & 197 \\
\hline
\end{tabular}

Note: Number of months defined as uncertain from 1960:1 - 2011:12 for Macro Uncertainty, 1985:1 - 2015:2 for Policy Uncertainty, 2000:1 -2014:12 for State Uncertainty and 1990:1 - 2015:2 for the VIX; the $\alpha$ one-tailed significance level is from the Normal Distribution and the series assume to follow i.i.d. as in Bloom (2009).

we use the corresponding percentiles at various levels in our analysis to show the robustness of empirical results as well as to avoid the Normal i.i.d. assumption. Figure 3 shows the time periods defined as uncertain using different uncertainty proxies. The right-lower panel also displays the State Uncertainty proxy after aggregating, although there is substantial variation across states. Note, however, the similarities between the Policy Uncertainty indicator and our State Uncertainty proxy.

Figure 3 here

\section{$3 \quad$ Empirical Model and Results}

\subsection{Regression Model}

Our empirical model is given by

$$
y_{i t}=x_{i t-\tau} \vec{\gamma}+1_{u n c, i t-\tau} \vec{\beta}_{1 t-\tau}+\operatorname{Bartik}_{i t-\tau} \vec{\beta}_{2 t-\tau}+1_{u n c, i t-\tau} \times \operatorname{Bartik}_{i t-\tau} \vec{\beta}_{3 t-\tau}+\alpha_{i}+u_{i t}
$$

where $x_{i t-\tau}$ is a vector containing up to $\tau$ lags of the control variables, $\gamma$ is the corresponding parameter vector, $\alpha_{i}$ is the state specific intercept, $1_{u n c, i t-\tau}$ and $B a r t i k_{i t-\tau}$ are $(1 \times \tau)$ vectors of lagged uncertainty indicators and labor demand shocks, respectively, and $\vec{\beta}_{j t-\tau}, j=1,2,3$ 
are the corresponding $(\tau \times 1)$ parameter vectors. An element of $\vec{\beta}_{j t-\tau}$ reflects the impact of the respective lag, while the sum of the elements gives the long-run impact. We experimented with different lag-lengths and use $\tau=6$ lags as baseline specification, but the results are not sensitive to the number of lags as long as we use more than two and less than seven. The (sum of the elements in the) coefficient vectors of main interest are $\vec{\beta}_{1}, \vec{\beta}_{2}$ and $\vec{\beta}_{3} \cdot \vec{\beta}_{1}$ reflects the impact of a regime-change from low to high uncertainty, $\vec{\beta}_{2}$ reflects the impact of a local labor demand shock on the housing market and $\vec{\beta}_{3}$ states the (change in the) effect of a local labor demand shock in a period of high uncertainty. In other words, $\vec{\beta}_{3}$ is a measure for the change in the responsiveness of the housing market variables due to high uncertainty. If $\vec{\beta}_{3}$ is significantly different from zero and its sign is different (same) from $\vec{\beta}_{2}$, then uncertainty diminishes (amplifies) the impact of the local labor demand shock.

For example, in an uncertain period, even though the impact of an adverse labor demand shock on the housing price is negative, home sellers will most likely not sell at the lower prices as this would unnecessarily reduce the return of the most important asset of most households. The underlying assumption is that the investment opportunity (selling or buying the house) is irreversible once exercised but available until then. In that sense, $\vec{\beta}_{3}$ proxies the real option value by capturing the change in the equilibrium housing price or the median selling price that does not materialize following a labor demand shock because of uncertainty.

Before we empirically investigate the role of uncertainty - and labor demand shocks in the housing market, we first address various econometric issues in our empirical setup. First, to account for spatial dependence, heterogeneity and autocorrelation, we use the standard errors developed in Driscoll and Kraay (1998). Second, to address endogeneity issue, we perform Durbin-WuHausman endogeneity tests. Table 2 shows the $p$-values for the specific lag of Macro Uncertainty using the first six lags of the Bartik index as exogenous variables in the reduced forms. ${ }^{11}$ The null

\footnotetext{
${ }^{11}$ For example, the $p$-value from the column Lag 1 is computed as follows. First, the first lag of Macro Uncertainty is regressed on the first six lags of all control variables and the Bartik index, and the residual $v_{1 i t}$ from this estimation is stored. Second, $v_{1 i t}$ is included into the estimation of equation (2) but without including the Bartik index and the interaction term. The $p$-value of the coefficient of $v_{1 i t}$ is displayed in the Table 2 . In addition, a joint significance
} 
hypothesis of exogeneity of Macro Uncertainty cannot be rejected.

Table 2: Endogeneity Test for Macro Uncertainty.

\begin{tabular}{l|c|c|c|c|c|c|c|}
\hline \hline Lag & 1 & 2 & 3 & 4 & 5 & 6 & Joint test \\
Macro Uncertainty & 0.224 & 0.179 & 0.777 & 0.828 & 0.545 & 0.402 & 0.261 \\
\hline
\end{tabular}

Note: The Table displays the $p$-values of Durbin-Wu-Hausman endogeneity tests for the specific lag of Macro Uncertainty using the first six lags of the Bartik index as exogenous variables in the reduced forms.

To further guard against possible simultaneous effects, we lag all the explanatory variables in equation (2). Moreover, by construction, our uncertainty measure are exogenous. For example, our benchmark Macro Uncertainty measure, as stated above, consists of purely unforecastable components. Consequently, by the definition and construction of the Macro Uncertainty measure, there should not be any underlying simultaneity between housing market variables and the Macro Uncertainty. Moreover, the VIX, which captures the expected volatility of the S\&P 500 index, is also unlikely to be strongly influenced by housing prices. And, although, Policy Uncertainty and the State Uncertainty measure might be affected in the same period news, it seems rather unlikely that housing prices today affect yesterday's news coverage. Additionally, we include a rich set of controls to avoid an omitted variable bias. ${ }^{12}$ As for the Bartik index, the local labor demand shocks $B a r t i k_{i t}$ are constructed to be exogenous given a constant labor supply. Binary uncertainty indicators are coded to be one if uncertainty is above a threshold value and zero otherwise.

\subsection{Baseline Results}

Our empirical objectives are to show (i) the quantitative effect of uncertainty on the housing market, (ii) the change in the impact of local labor demand shocks on the housing market if they occur during periods of uncertainty, and (iii) how robust are the outcomes given the choice of the uncertainty proxy and the threshold level defining a period of high uncertainty? Table 3 shows the

test of all six residuals from the estimations of the six lags of Macro Uncertainty is presented in the last column.

12 In particular, due to the long time dimension, we cannot use time fixed-effects in this setting. Therefore, we include a host of controls in order to capture variation in the economic environment. The complete set of control variables used for our empirical analysis is shown in the Appendix. 
estimation results for equation (2). The second (Regression model 1) and third (Regression model 2) columns show the individual effects of Macro Uncertainty - and labor demand shocks, while the columns 4 to 6 (Regression model 3) include both shocks as well as the interaction term. ${ }^{13}$

Table 3: Long-run Effects of Uncertainty, Bartik and Interaction term

\begin{tabular}{|c|c|c|c|c|c|c|}
\hline & $\begin{array}{l}\text { Regression } \\
\text { Model } 1\end{array}$ & $\begin{array}{l}\text { Regression } \\
\text { Model } 2\end{array}$ & $\begin{array}{l}\text { Regressic } \\
\text { Model } 3\end{array}$ & & & \\
\hline Dependent Variable & $1_{\text {macro }}($ no Bartik) & Bartik (no $1_{\text {macro }}$ ) & $1_{\text {macro }}$ & Bartik & Bartik*1 $1_{\text {macro }}$ & Obs. \\
\hline$\Delta \log ($ median sales price $)$ & $\begin{array}{l}-.0168^{* *} \\
(.00726)\end{array}$ & $\begin{array}{l}16.142^{*} \\
(8.3599)\end{array}$ & $\begin{array}{l}-.0180 * * \\
(.00752)\end{array}$ & $\begin{array}{l}32.63^{* * *} \\
(10.679)\end{array}$ & $\begin{array}{l}-31.68^{* * *} \\
(11.765)\end{array}$ & 6,539 \\
\hline$\Delta \log ($ house price $)$ & $\begin{array}{l}-.0131^{* * *} \\
.00367\end{array}$ & $\begin{array}{l}6.3675^{*} \\
(3.7228)\end{array}$ & $\begin{array}{l}-.0142^{* * *} \\
(.00344)\end{array}$ & $\begin{array}{l}10.93^{* * *} \\
(3.8337)\end{array}$ & $\begin{array}{l}-14.35^{* * *} \\
(4.3892)\end{array}$ & 13,158 \\
\hline$\Delta \%$ selling for loss & $\begin{array}{l}.5392 \\
(.37492)\end{array}$ & $\begin{array}{l}-692.653^{*} \\
(379.579)\end{array}$ & $\begin{array}{l}.52575 \\
(.37032)\end{array}$ & $\begin{array}{l}-1133.00^{* *} \\
(492.26)\end{array}$ & $\begin{array}{l}994.94^{* *} \\
(485.88)\end{array}$ & 5,904 \\
\hline$\Delta$ turnover & $\begin{array}{l}.0159 \\
(.05597)\end{array}$ & $\begin{array}{l}37.278 \\
(33.334)\end{array}$ & $\begin{array}{l}-.0036 \\
(.05451)\end{array}$ & $\begin{array}{l}147.26^{* *} \\
(66.317)\end{array}$ & $\begin{array}{l}-202.00^{* *} \\
(79.781)\end{array}$ & 6,011 \\
\hline
\end{tabular}

Note: Sample period from 1990 onwards. The long-run effects of uncertainty (95th percentile threshold), Bartik and interaction term are presented with corresponding standard errors in brackets. ${ }^{*}$ indicates significance at $10 \%$ level, ${ }^{* *}$ indicates significance at $5 \%$ level, *** indicates significance at $1 \%$ level

Regression model 1 shows the long-run impact, $\vec{\beta}_{1}$, of Macro Uncertainty on the changes in log median sale prices, changes in log housing prices, changes in the percentage houses selling for loss, and changes in turnover (housing transactions). For all three regression models, we control for the federal funds rate, housing starts as a proxy for residential investment, income, industrial production, inflation, population, and the S\&P 500 and the unemployment rate. We find that uncertainty adversely affects the median sale prices and house prices on average by $1.68 \%$ and $1.31 \%$, respectively. In other words, Dorofeenko et al. (2014) results are driven by the supply side $^{14}$, which our empirical results do not necessarily support. Moreover, we find uncertainty impacts neither turnover nor the share of houses selling for loss directly. The intuition for this findings is that in the long-run uncertainty decreases, on average, buyers' willingness to pay at the asking price. This, in turn, leads sellers to reduce the asking price which reduces the equilibrium

\footnotetext{
13 We use 95th percentile as our cut off point for Macro Uncertainty. We also estimate analogous regression using State Uncertainty and VIX uncertainty measures. The results from other regressions are similar to Macro Uncertainty. The complete regressions results are in Appendix, Table 13.

14 Dorofeenko et al. (2014) show that an increase in their measure of uncertainty has an increasing effect on house prices due to the default premium on the housing developers: There is a markup on housing prices due to the bankruptcy possibility that is caused by uncertainty.
} 
housing price. Regression model 2 shows the long-run impact of labor demand shocks, $\vec{\beta}_{2}$, proxied by the Bartik index. A positive labor demand shock all leads to the expected sign on the housing variables. All the housing variables, except for the Turnover, are statistically significant at the $10 \%$ level.

Turning now to the full scale regression model 3 , as in model 1, we again find that Macro Uncertainty adversely affects the median sale prices and house prices on average by $1.80 \%$ and $1.42 \%$, respectively. But the effects are statistically insignificant for the loss for sale and the turnover. For the Bartik index (labor demand shock effect), the impact is highly statistically significant for all dependent variables, even after controlling for state-level unemployment. For example, one standard deviation increase in the local labor demand shock, increases house prices, median sale prices and transactions on average by $.14 \%, .43 \%$ and $1.92 \%$-points, respectively and decreases the share of houses selling for loss by $14.77 \%$-points. Due to linearity, the signs reverse in the case of adverse labor demand shocks - as observed in most states during the Great Recession period. ${ }^{15}$ The Bartik index effect is twice as large as the results from model 2. The reason is that in the model 2, the Bartik index captures the uncertainty effects that are absent. In the full regression model 3, the interaction term, $\vec{\beta}_{3}$, mitigates the marginal Bartik effect in periods of high uncertainty. Therefore, not accounting for Macro Uncertainty may lead to biased estimates of the effects of labor demand shocks.

For the robustness check on the uncertainty measures, we also show the results for different threshold values (i.e. percentile cutoffs) as shown in Figure 4. Regardless of the threshold value, the sign and the significance of the estimated $\vec{\beta}_{1}$ for the log house price and log median sales price do not change. ${ }^{16}$

Figure 4 here

\footnotetext{
${ }^{15}$ We report the impact of a standard deviation increase due to the scale of the bartik. Mean local labor demand decreases from 1990 until 2014 by $0.004 \%$-points, while one standard deviation corresponds to 0.013\%-points: For example, for the log house price, we report an increase of $0.14 \%$ as $0.013 \times 10.93$, while the real option value is calculated similarly as $0.013 \times 14.35=0.19 \%$, where $\beta_{3}=14.35$.

16 All of the coefficients are significant at a $1 \%$ significance level, except for one which is significant at the $5 \%$ level.
} 
The above results indicate that the uncertainty and labor demand shocks affect the housing market variables in opposite direction. To determine the quantitative effects of these two shocks on the housing variables, we further analyze the interaction term, $\vec{\beta}_{3}$ : the results are shown in the sixth column of Table 3. When the labor demand shock occurs during a period of high uncertainty, for almost every dependent variable and threshold level, the effect of uncertainty shock dominates the labor demand shock: a clear sign change from the estimated $\vec{\beta}_{2}$ being positive to the estimated $\vec{\beta}_{3}$ being negative. To quantify $\vec{\beta}_{3}$ effect as the homeowners' diminished response ("wait and see effect") following a labor demand shock, our empirical results show a decrease of $0.19 \%(0.013 \% \times 14.35)$ of the house price and a decrease of $0.41 \%(0.013 \% \times 31.68)$ of the median sale price. For the expositional purpose of the interaction term, Figure 5 shows the effects of a labor demand shock with - and without uncertainty shock (using our benchmark Macro Uncertainty shock). The blue line (Bartik Normal Times) summarizes the long-run impact of labor demand shocks, $\vec{\beta}_{2}$, on the various dependent variables, while the red line (Bartik High Uncertainty) represents the impact of labor demand shocks in uncertainty times, i.e. $\vec{\beta}_{2}+\vec{\beta}_{3}$. Figure 5 clearly shows that when uncertain periods occur then the effect of the labor demand shock is greatly muted. These dominating uncertainty shock effects suggest the presence of real options effects in housing market. ${ }^{17}$ Figure 6 is analogous to Figure 5, but with the State Uncertainty shock: the results are not overturned.

Figures 5 and 6 here

Overall, we find that the results in Bloom et al. (2007) for the firm level carry over to the housing market: uncertainty greatly diminishes the responsiveness of housing market variables. We note, however, our results are somewhat sensitive to the choice of the uncertainty proxy, which can be seen in Table 13 in Appendix. ${ }^{18}$

\footnotetext{
17 This result is in line with the findings of Davis and Quintin (2014), who find that uncertainty about housing prices kept the default rate low relative to a situation without uncertainty.

18 Although we do not show the results with the Policy Uncertainty shock in Table 13, the real options effects $\left(\vec{\beta}_{3}\right)$ from the Policy Uncertainy are not as strongly associated if high threshold values (90th or 95th percentile) are used. The reason might be that when the 95th percentile threshold, the Policy Uncertainty proxy represents only the periods that are associated with the post 2011 period (this includles the period during the European Debt
} 


\subsection{Grouping States by Housing Price Volatility}

To analyze whether the real option effect varies by regions, we sort the fifty U.S. states into three groups according to the unconditional housing price volatility in each state over time, and we estimate our model (2) for each one of the groups. The three groups are equal size and we refer to them as low, medium and high: Our hypothesis is to test empirically whether the change in the responsiveness of housing market variables is larger in the states with higher housing price volatilities compared to the lower housing price volatilities states. Consequently, we focus on the dominant effect of State Uncertainty over the labor demand shocks for each one of the groups, using the 95th percentile of the State-level uncertainty proxy. We choose the State-level uncertainty measure because we group the states according to the state-specific housing price volatility; the results are qualitatively identical, however, for the Macro Uncertainty measure. Table 4 shows the results for the three different groups.

Table 4: Long-run Effects of Bartik and Interaction term grouped by the magnitude of the housing price volatility over time.

\begin{tabular}{|c|cc|cc|cc|}
\hline \hline Housing Price Volatility & \multicolumn{2}{|c|}{ low } & \multicolumn{2}{c|}{ medium } & \multicolumn{2}{c|}{ high } \\
\hline \hline & Bartik (B) & $\mathrm{B}^{*} 1_{\text {state }}^{\text {low }}$ & $\mathrm{B}$ & $\mathrm{B}^{*} 1_{\text {state }}^{\text {medium }}$ & $\mathrm{B}$ & $\mathrm{B}^{*} 1_{\text {state }}^{\text {high }}$ \\
\hline \hline$\Delta \log ($ house price) & $18.47^{* *}$ & -6.85 & $7.055^{* * *}$ & -9.26 & $21.26^{* * *}$ & $-25.0^{* * *}$ \\
& $(7.802)$ & $(7.131)$ & $(2.596)$ & $(6.253)$ & $(6.899)$ & $(8.905)$ \\
\hline
\end{tabular}

Note: The long-run effects of Bartik and interaction term based on State Uncertainty (95th percentile threshold) are presented with corresponding standard errors in brackets grouped by housing price volatility across states. ${ }^{*}$ indicates significance at $10 \%$ level, ${ }^{* *}$ indicates significance at $5 \%$ level, ${ }^{* * *}$ indicates significance at $1 \%$ level.

The most striking difference between the three groups is with respect to the significance and the magnitude of our responsiveness measure $\left(\vec{\beta}_{3}\right)$ for the high group. As one moves away from the low to high volatility group, the interaction term $\left(\vec{\beta}_{3}\right)$ not only increases in absolute magnitude from -6.85 to -25 but also becomes highly statistically significant. That is, the effect of a one standard deviation increase (i.e. $0.013 \%$-points) in the interaction term changes from $-6.85 \times 0.013=$

crisis). And hence, there is not enough sample size to test for the interaction terms. However, if the 85th percentile is taken as threshold value, the interaction effects become significant again, as more periods, especially the months before 2010, are classified as periods of high uncertainty. 
$0.09 \%$ in the low group to $-25.0 \times 0.013=0.32 \%$ of the housing price in the high group.

\subsection{Grouping States by the Impact of Local Labor Demand Shocks}

For the robustness check, we also sort groups by the impact of local labor demand shocks. We calculate the impact of the Bartik index based on our model (2) with housing prices as dependent variable, but estimating time-series regressions for each state. We include states where the Bartik has a significant impact ( $5 \%$ level) on the change in log housing prices, which results in 37 states. We sort these 37 states into three groups of almost equal size, depending on the magnitude of the Bartik's impact. Table 5 shows the long-run effects of the Bartik and the interaction term. By construction, the impact of the Bartik increases and is highly significant. The interaction

term, however, is only statistically significant for the group high, with the sum of $\widehat{\vec{\beta}}_{2}$ and $\widehat{\vec{\beta}}_{3}$ $($ e.g.104.9 $-102=2.9)$ very close to zero: the net effect on the change in log housing prices is almost zero. That is, in times of high uncertainty, home sellers and -buyers do not trade at the price and wait out until the uncertainty periods are over. Moreover, an explanation for the dominance of uncertainty over the shock for the high group, in contrast to the medium and low group, is that the larger the impact of the shock, the less responsive households are, ceteris paribus.

Table 5: Long-run Effects of Bartik and Interaction term grouped by the impact of the bartik in each State.

\begin{tabular}{l|cc|cc|cc|}
\hline \hline Bartik Index & \multicolumn{2}{|c|}{ low } & \multicolumn{2}{c|}{ medium } & \multicolumn{2}{c|}{ high } \\
\hline \hline & Bartik (B) & $\mathrm{B}^{*} 1_{\text {State }}^{\text {low }}$ & $\mathrm{B}$ & $\mathrm{B}^{*} 1_{\text {State }}^{\text {medium }}$ & $\mathrm{B}$ & $\mathrm{B}^{*} 1_{\text {State }}^{\text {high }}$ \\
\hline \hline$\Delta \log ($ house price) & $9.835^{* * *}$ & -5.16 & $52.98^{* * *}$ & -16.1 & $104.9^{* * *}$ & $-102^{* *}$ \\
& $(2.328)$ & $(5.947)$ & $(9.703)$ & $(14.43)$ & $(21.13)$ & $(45.07)$ \\
\hline
\end{tabular}

Note: The long-run effects of Bartik and interaction term based on State Uncertainty (95th percentile threshold) are presented with corresponding standard errors in brackets grouped by housing price volatility across states. ${ }^{*}$ indicates significance at $10 \%$ level, ${ }^{* *}$ indicates significance at $5 \%$ level, ${ }^{* * *}$ indicates significance at $1 \%$ level. 


\subsection{Robustness Checks}

Our empirical results are robust to a variety of alternative specifications, such as including a recession dummy, using different lag lengths, constructing the Bartik index following Charles, Hurst and Notowidigdo (2013), scaling the State Uncertainty indicator by the number of newspapers and normalizing it by dividing by the standard deviation in each state, or omitting some of the variables from the vector of controls variables. However, the results are not robust to omitting the Great Recession period, i.e. using the sample from 1990:1 until 2007:12. This may not be too surprising in light of Figure 3, which shows a lot of the variation in the uncertainty dummy comes from the differences between the time before and after 2008 .

\section{Conclusion}

Our empirical results lend support for the real option effects in the U.S. housing market and are in line with some of the predictions of Bloom et al.'s (2007) theoretical model. Using the statelevel panel data from 1990:1 to 2014:12, we show (i) Macro Uncertainty has a small but highly significant impact on the level of housing prices but not on quantities, (ii) Macro Uncertainty dominates the effects of (adverse) labor demand shocks and (iii) the results are robust to changes in the threshold defining times of high uncertainty. We interpret this result as the different proxies capturing different aspects of uncertainty, with the proxy of Jurado et al. (2015) being well suited, due to its construction, to capture the spells of uncertainty that induce macro-level real options effects. These findings might be helpful for housing policy makers to mitigate adverse effects of real shocks on housing markets during periods of high uncertainty before they materialize. 


\section{Bibliography}

1. Aastveit, K. A., Natvik, G. J., \& Sola, S. (2013). Economic uncertainty and the effectiveness of monetary policy. Norges Bank mimeo.

2. Baker, S. R., Bloom, N., \& Davis, S. J. (2016). Measuring economic policy uncertainty. Quarterly Journal of Economics, forthcoming.

3. Bartik, T. (1991). Who benefits from state and local economic development policies? Kalamazoo: W.E. Upjohn Institute for Employment Research.

4. Berger, T., Grabert, S. and Kempa, B. (2017). Global macroeconomic uncertainty. Journal of Macroeconomics, 53, 42-56.

5. Bloom, N. (2009). The impact of uncertainty shocks. Econometrica, 77 (3), 623-685.

6. Bloom, N., Bond, S. and Van Reenen, J. (2007). Review of Economic Studies, (74), 391-415.

7. Bloom, N. (2014). Fluctuations in uncertainty. Journal of Economic Perspectives, 28 (2), $153-76$.

8. Bulan, L., Mayer, C. \& Somerville, C. T. (2009). Irreversible investment, real options, and competition: evidence from real estate development. Journal of Urban Economics. 65, 237-251.

9. Capozza D. \& Helsley H. (1989). The stochastic city. Journal of Urban Economics, 26, 295 -306 .

10. Charles, K., Hurst, E., \& Notowidigdo, M. (2013). Manufacturing decline, housing booms, and non-employment. Chicago Booth mimeo.

11. Childs, P., Riddiough, T. \& Triantis, A. (1996). Mixed uses and the redevelopment option. Real Estate Economics, 24 (3), 317-339. 
12. Childs, P., Ott, S. \& Riddiough, T. (2002). Real Estate Economics, 30 (3), 415-443.

13. Christiano, L. J., Motto, R., \& Rostagno, M. (2014). Risk shocks. American Economic Review, 104 (1), 27-65.

14. Clapp, J. M., Eichholtz, P. \& Lindenthal, T. (2013). Real option value over a housing market cycle. Regional Science and Urban Economics, 43 (6), 841-1040.

15. Cunningham, C. R. (2006). House price uncertainty, timing of development, and vacant land prices: evidence for real options in Seattle. Journal of Urban Economics, 59 (1), 1-31.

16. Cunningham, C. R. (2007). Growth controls, real options, and land development. The Review of Economics and Statistics, 89 (2), 343-358.

17. Davis, M. A., \& Quintin, E. (2014). Default when current house prices are uncertain. University of Wisconsin-Madison mimeo.

18. Dorofeenko, V., Lee, G. S., \& Salyer, K. D. (2014). Risk shocks and housing supply: A quantitative analysis. Journal of Economic Dynamics and Control , 45, 194-219.

19. Driscoll, J. C., \& Kraay, A. C. (1998). Consistent covariance matrix estimation with spatially dependent panel data. Review of Economics and Statistics, 80 , 549-560.

20. Edlund, L., Machado, C., \& Sviatschi, M.M. (2016). Bright minds, big rent: gentrification and the rising returns to skill. NBER Working Paper No. 21729.

21. Holland, S., Ott, S. \& Riddiough, T. (2000). The role of uncertainty in investment: An examination of competing investment models using commercial real estate data. Real Estate Economics, 28 (1), 33-64.

22. Jurado, K., Ludvigson, S., \& Ng, S. (2015). Measuring uncertainty. American Economic Review, 105 (3), 1177-1216. 
23. Mehkari, M. S. (2016). Uncertainty shocks in a model with mean-variance frontiers and endogenous technology choices. Journal of Macroeconomics, 49, 71-98.

24. Saks, R. E. (2005). Job creation and housing construction: constraints on metropolitan area employment growth. Finance and Economics Discussion Series No. 2005-49 Board of Governors of the Federal Reserve System.

25. Shoag, D., \& Veuger, S. (2016). Uncertainty and the geography of the great recession. Journal of Monetary Economics, 84, 84-93.

26. Strobel, J. (2015). On the different approaches of measuring uncertainty shocks. Economics Letters, $134,69-72$.

27. Stroebel, J., \& Vavra, J. (2015). House prices, local demand, and retail prices. Kilts Center for Marketing at Chicago Booth - Nielsen Dataset Paper Series 1-030. 


\section{Appendix}

\subsection{Data Description and Other Regressions}

Table 6: Uncertainty Proxies

\begin{tabular}{|l|lll|}
\hline \hline Variable & Availability & Source & Regional level \\
\hline Macro Uncertainty & 1960M1-2011M12 & Jurado et al. (2015) & National \\
Policy Uncertainty & 1985M1-2015M2 & Baker et al. (2012) & National \\
State Uncertainty & 2000M1-2014M12 & Self constructed & State \\
VIX & 1990M1-2015M2 & FRED & National \\
\hline \hline
\end{tabular}

Table 7: Dependent Variables

\begin{tabular}{|l|lll|}
\hline \hline Variable & Availability & Source & Regional level \\
House Price & 1975M1-2014M12 & Freddie\&Mac & State \\
Median Sales Price & 1996M4-2014M12 & Zillow Database & State \\
\% Selling For Loss & 1998M1-2014M12 & Zillow Database & State \\
Total Turnover & 1998M1-2014M12 & Zillow Database & State \\
\hline \hline
\end{tabular}

Table 8: Control Variables

\begin{tabular}{|l|lll|}
\hline \hline Variable & Availability & Source & Regional level \\
\hline Federal Funds Rate & 1954M7-2015M1 & FRED & State \\
Housing Starts & 1988M1-2015M1 & FRED & State \\
Income & 1950Q1-2014Q3 & BEA & State \\
Industrial Production & 1919M1-2015M1 & FRED & National \\
Inflation Rate & 1947M1-2015M1 & FRED & National \\
Population & 1972-2013 & FRED & State \\
S\&P 500 & 1970M1-2015M3 & Datastream & National \\
Unemployment Rate & 1976M1-2014M12 & FRED & State \\
\hline \hline
\end{tabular}


Table 9: Descriptive statistics of the housing market variables.

\begin{tabular}{|l|l|l|l|l|l|}
\hline \hline & Obs. & Mean & Std. Dev. & Min & Max \\
\hline house price & 24480 & 125.5488 & 25.6362 & 61.0220 & 275.6024 \\
$\Delta \log ($ house price) & 24429 & 0.0002795 & 0.0073951 & -0.1098976 & 0.0773649 \\
Median Sales Price & 7790 & 191184.8 & 74180.34 & 47519.08 & 518470.1 \\
$\Delta \log$ (Median Sales Price) & 7751 & 0.001178 & 0.025107 & -0.256864 & 0.308221 \\
\% Selling For Loss & 7234 & 12.8908 & 13.5806 & 0.0612 & 70.5068 \\
$\Delta \%$ Selling For Loss & 7158 & 0.107329 & 1.18954 & -15.6326 & 16.4346 \\
Turnover & 7308 & 4.81494 & 2.253468 & 0.008869 & 17.16583 \\
$\Delta$ Turnover & 7271 & 0.0032471 & 0.106966 & -12.71301 & 2.019346 \\
\hline \hline
\end{tabular}

Table 10: Descriptive statistics of the uncertainty measures as well as the bartik index.

\begin{tabular}{|l|l|l|l|l|l|}
\hline \hline & Obs. & Mean & Std. Dev. & Min & Max \\
\hline Macro Uncertainty & $264^{*} 51$ & 0.67773 & 0.0961123 & 0.568981 & 1.130619 \\
Policy Uncertainty & $300 * 51$ & 106.3401 & 34.38186 & 57.20262 & 245.1267 \\
State Uncertainty & 9180 & 18.23878 & 7.730284 & 0 & 233 \\
VIX & $300 * 51$ & 19.9604 & 7.730284 & 10.82 & 62.64 \\
Bartik & 15249 & -0.000041 & 0.0001304 & -0.002793 & 0.0009686 \\
\hline \hline
\end{tabular}

Table 11: Sorted states, according to their unconditional housing price volatility over time.

\begin{tabular}{ccc}
\hline \hline low & medium & high \\
\hline Alabama & Alaska & Arizona \\
Arkansas & Colorado & California \\
Georgia & Delaware & Connecticut \\
Iowa & Idaho & District of Columbia \\
Indiana & Illinois & Florida \\
Kansas & Louisiana & Hawaii \\
Kentucky & Maine & Massachusetts \\
Missouri & Michigan & Maryland \\
Mississippi & Minnesota & New Hampshire \\
North Carolina & Montana & New Jersey \\
Nebraska & North Dakota & Nevada \\
New Mexico & Oklahoma & New York \\
Ohio & Pennsylvania & Oregon \\
South Carolina & Texas & Rhode Island \\
South Dakota & Utah & Virginia \\
Tennessee & Vermont & Washington \\
Wisconsin & West Virginia & Wyoming \\
\hline \hline
\end{tabular}


Table 12: Sorted states, according to the impact of the bartik index in each state.

\begin{tabular}{ccc}
\hline \hline low & medium & high \\
\hline Colorado & Arkansas & Alaska \\
Georgia & Kansas & Arizona \\
Iowa & Massachusetts & District of Columbia \\
Illinois & Maryland & Delaware \\
Kentucky & Minnesota & Hawaii \\
Louisiana & Missouri & Maine \\
Michigan & North Dakota & New Hampshire \\
Mississippi & Nebraska & New Mexico \\
North Dakota & New Jersey & Oregon \\
New York & South Carolina & South Dakota \\
Oklahoma & Virginia & West Virginia \\
Tennessee & Washington & Wyoming \\
Texas & & \\
\hline \hline
\end{tabular}

Table 13: Long-run Effects of Uncertainty, Bartik and Interaction term: Other Uncertainty measures

\begin{tabular}{l|lll|lll}
\hline Dep. Variable & $1_{\text {State }}$ & Bartik $(\mathrm{B})$ & $\mathrm{B}^{*} 1_{\mathrm{State}}$ & $1_{\mathrm{vix}}$ & Bartik $(\mathrm{B})$ & $\mathrm{B}^{*} 1_{\mathrm{vix}}$ \\
\hline \hline$\Delta \log ($ med sales price) & -.0033 & $30.296^{* * *}$ & $-24.84^{* *}$ & -.0058 & $42.316^{* * *}$ & $-44.64^{* * *}$ \\
& $(.00405)$ & $(11.723)$ & $(12.330)$ & $(.00930)$ & $(12.339)$ & $(16.513)$ \\
$\Delta \log ($ house price) & $-.0048^{* * *}$ & $15.315^{* * *}$ & $-17.63^{* * *}$ & .00191 & $12.625^{* * *}$ & -11.40 \\
& $(.00144)$ & $(4.2199)$ & $(4.4932)$ & $(.00482)$ & $(4.2128)$ & $(7.1745)$ \\
$\Delta \%$ selling for loss & $.48216^{* *}$ & $-1229 . *^{* *}$ & $1038.6^{*}$ & .48033 & $-1584.0^{* * *}$ & $1517.5^{* *}$ \\
& $(.23001)$ & $(479.62)$ & $(558.01)$ & $(.54268)$ & $(524.17)$ & $(699.86)$ \\
$\Delta$ turnover & $-.0577^{* * *}$ & $81.225^{*}$ & $-152.3^{* * *}$ & $.05951^{*}$ & $95.007^{*}$ & -102.4 \\
& $(.02065)$ & $(43.376)$ & $(57.010)$ & $(.03517)$ & $(54.964)$ & $(98.765)$ \\
\hline
\end{tabular}

Note: As the months defined as high uncertainty differ across the proxies, the variation used to identify $\vec{\beta}_{1 t-\tau}$ and $\vec{\beta}_{3 t-\tau}$, the coefficients of uncertainty and the interaction term, differs as well. The long-run effects of uncertainty (95th percentile threshold), Bartik and interaction term are presented with corresponding standard errors in brackets. * indicates significance at $10 \%$ level, $* *$ indicates significance at $5 \%$ level, ${ }^{* * *}$ indicates significance at $1 \%$ level. We do not include Policy Uncertainty by Baker et al. (2016) as the results similar to other measures and due to the space limitation. 
Figure 1: House Price Growth Rates and Uncertainty Proxies.

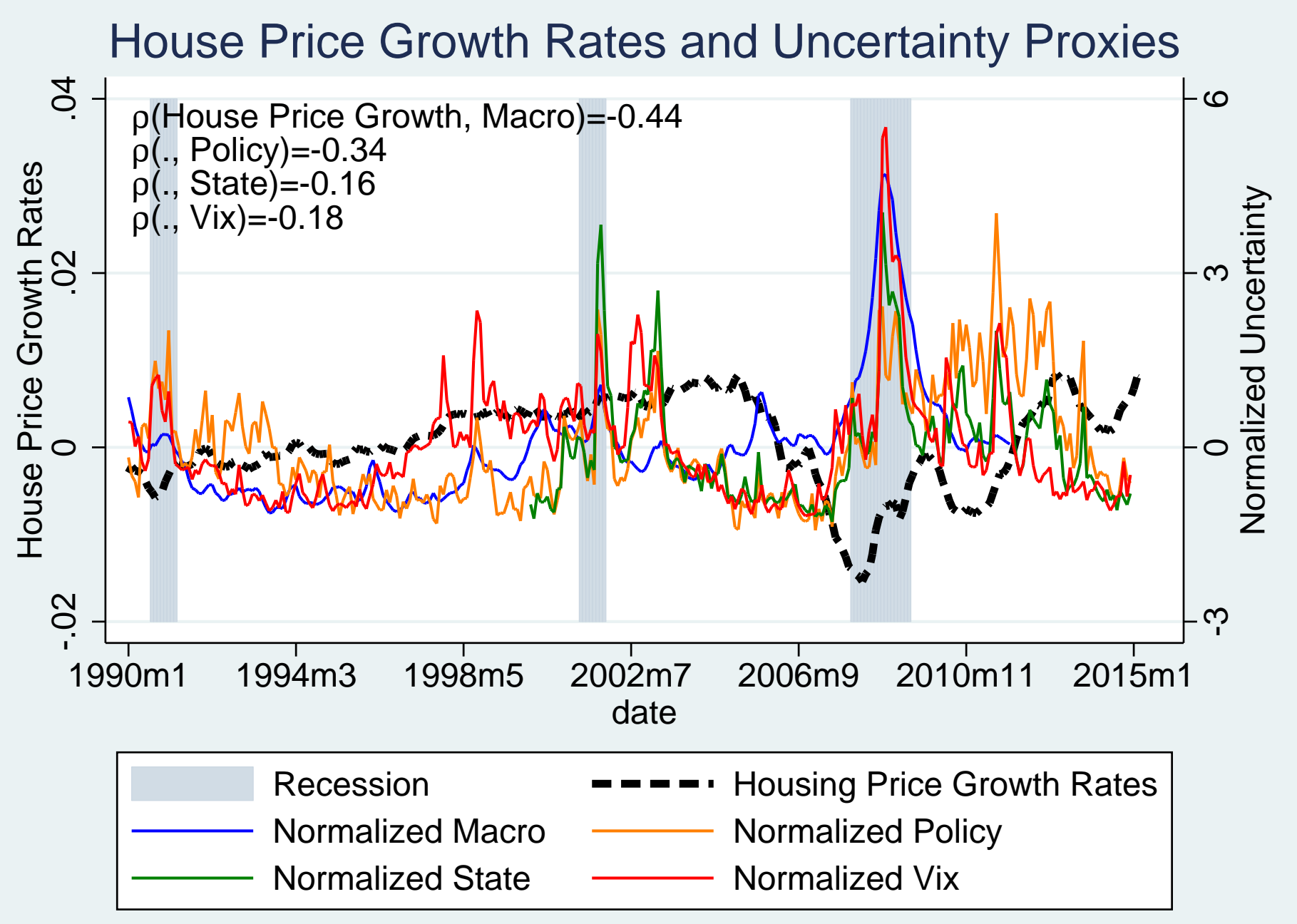


Figure 2: U.S. Unemployment Rate and Bartik Index.

\section{U.S. Unemployment Rate and Bartik Index}

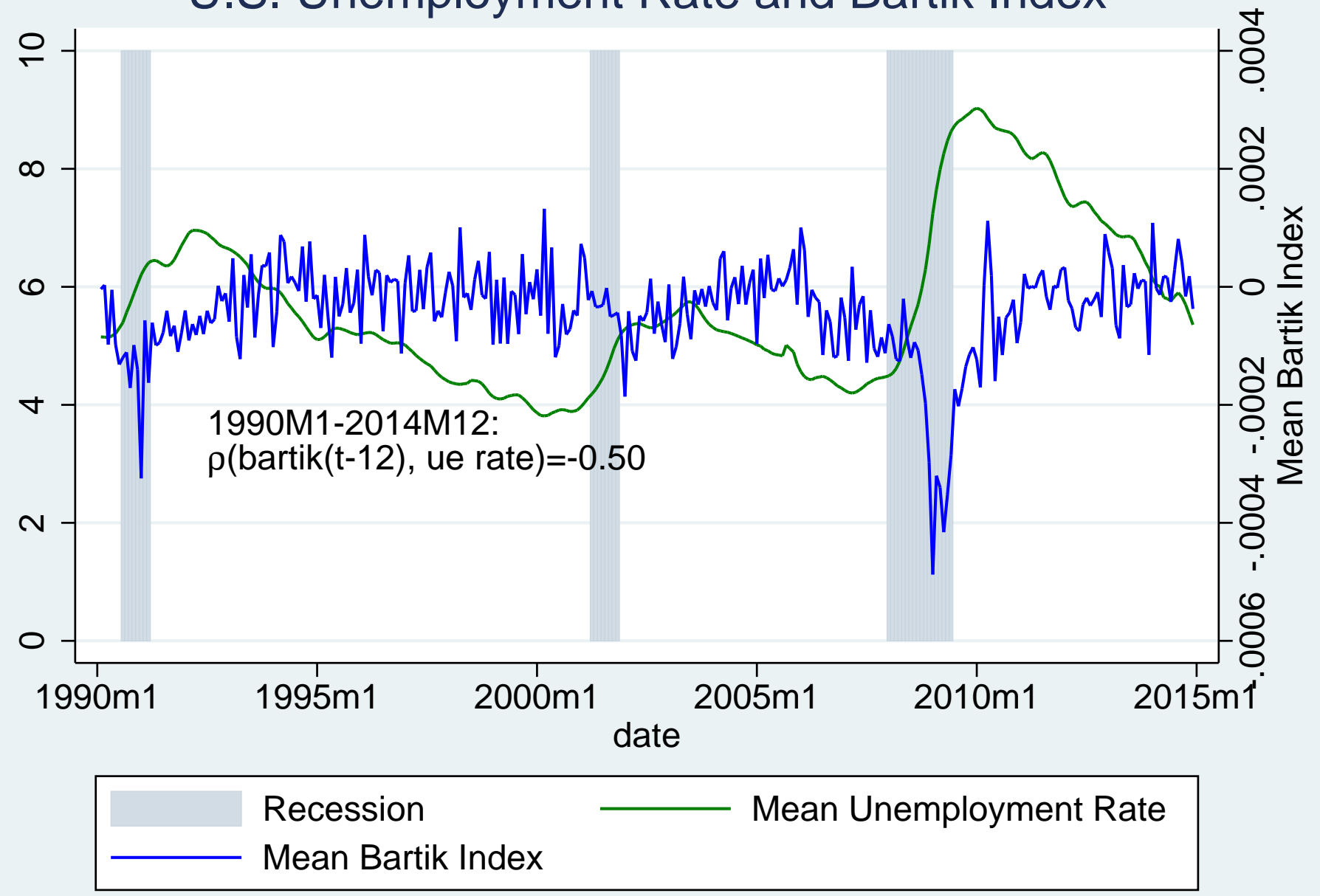


Figure 3: Periods of high uncertainty for different uncertainty proxies.
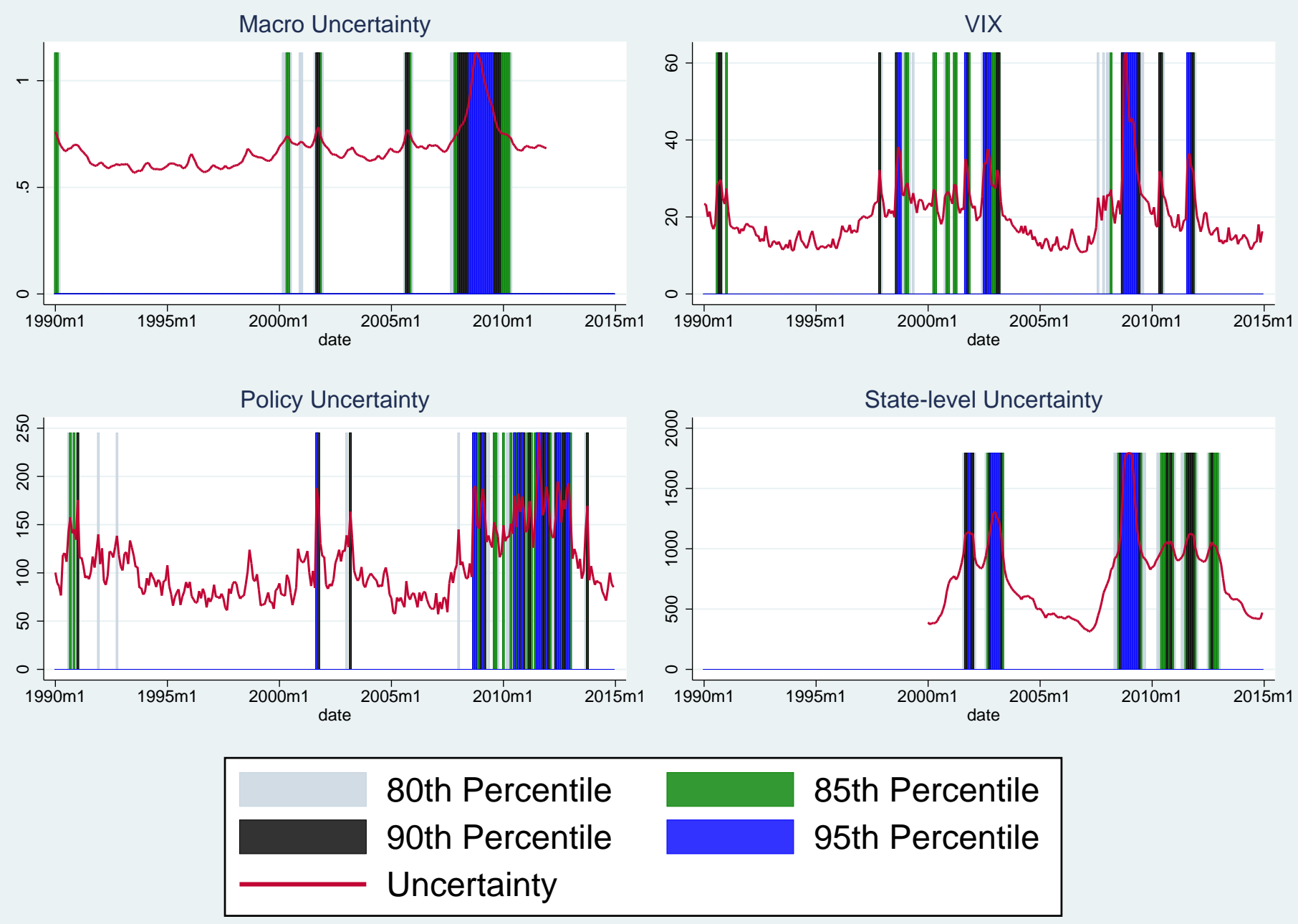
Figure 4: Impact of Macro Uncertainty.

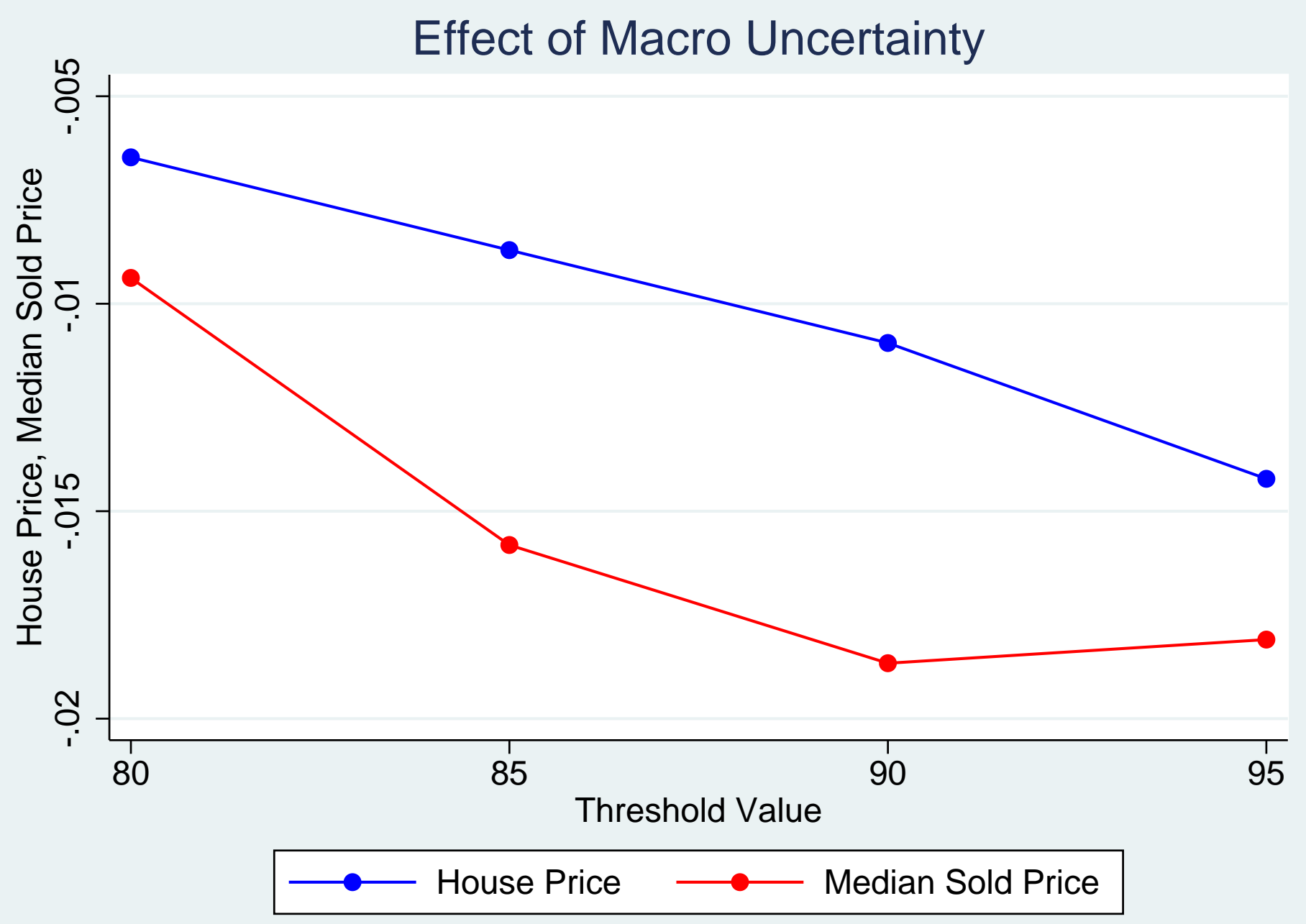


Figure 5: Impact of Bartik and Macro Uncertainty.

\section{Impact of Bartik and Macro Uncertainty}

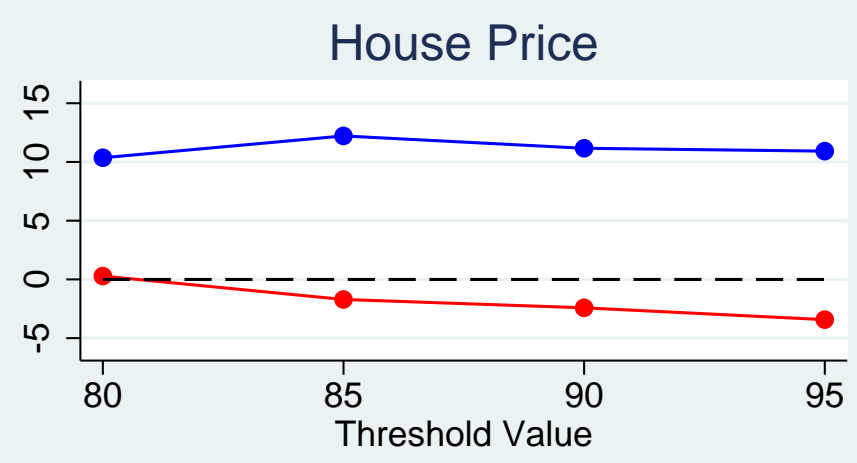

Median Sold Price

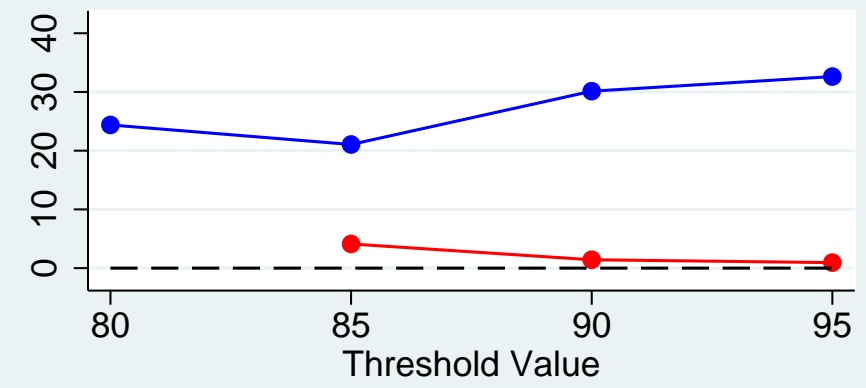

Percentage Selling For Loss
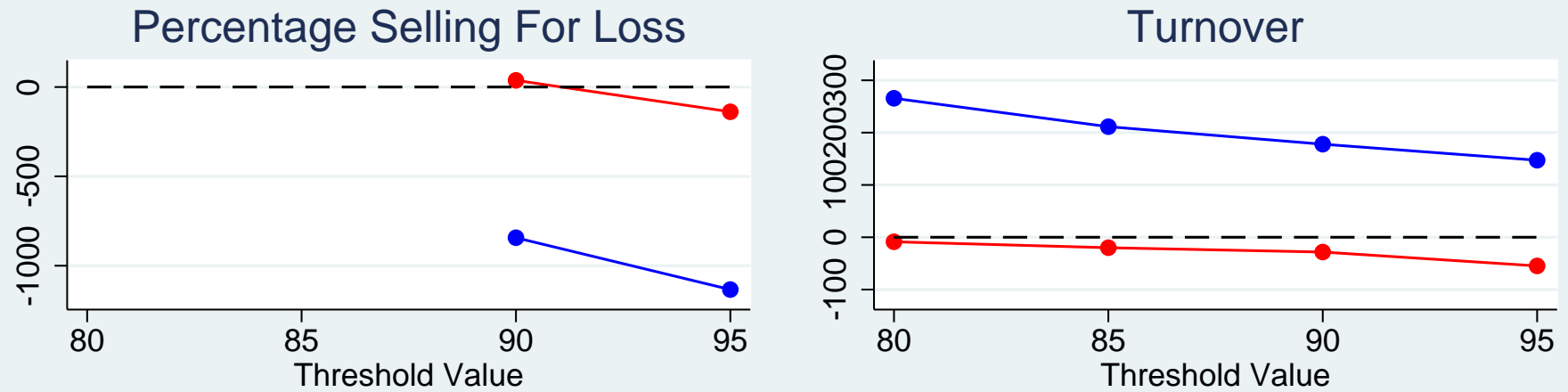

Bartik Normal Times

Bartik High Uncertainty 
Figure 6: Impact of Bartik and State Uncertainty.

\section{Impact of Bartik and State Uncertainty}
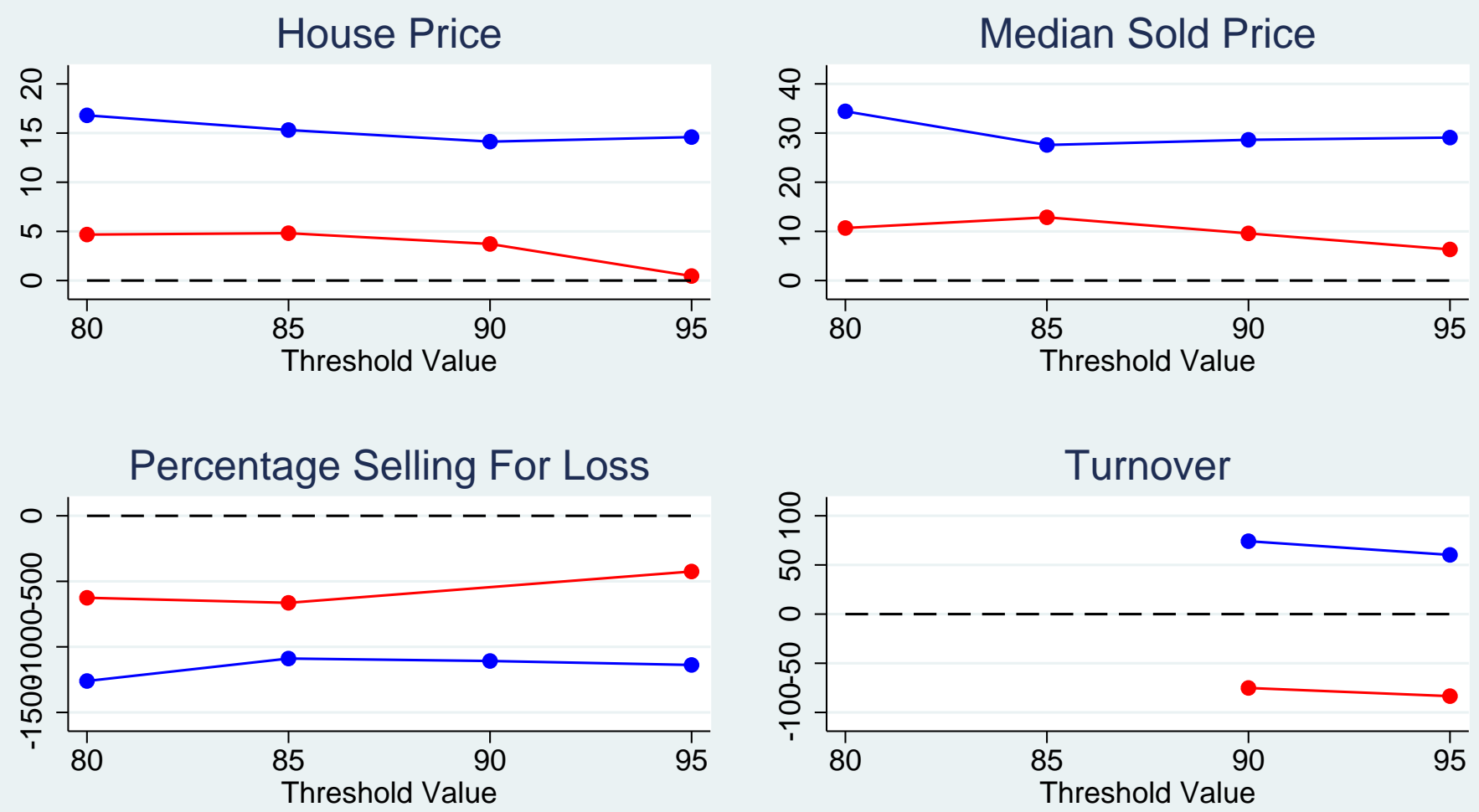

\section{Bartik Normal Times}

Bartik High Uncertainty 